Coulomb drag in parallel quantum dots

This content has been downloaded from IOPscience. Please scroll down to see the full text. 2009 EPL 8667004

(http://iopscience.iop.org/0295-5075/86/6/67004)

View the table of contents for this issue, or go to the journal homepage for more

Download details:

IP Address: 139.179.2.116

This content was downloaded on 15/07/2014 at 11:46

Please note that terms and conditions apply. 


\title{
Coulomb drag in parallel quantum dots
}

\author{
V. Moldoveanu ${ }^{1}$ and B. TanataR ${ }^{2}$ \\ ${ }^{1}$ National Institute of Materials Physics - P.O. Box MG-7, 077125 Bucharest-Magurele, Romania, EU \\ ${ }^{2}$ Department of Physics, Bilkent University - Bilkent, 06800 Ankara, Turkey
}

received 26 March 2009; accepted in final form 2 June 2009

published online 9 July 2009

PACS 73.23.Hk - Coulomb blockade; single-electron tunneling

PACS 85.35.Ds - Quantum interference devices

PACS 85.35.Be - Quantum well devices (quantum dots, quantum wires, etc.)

\begin{abstract}
We study theoretically the electronic transport in parallel few-level quantum dots in the presence of both intradot and interdot long-range Coulomb interaction. Each dot is connected to two leads and the steady-state currents are calculated within the Keldysh formalism using the random-phase approximation for the interacting Green functions. Due to the momentum transfer between the two systems it is possible to get a nonvanishing current through an unbiased Coulombblockaded dot, if the other dot is set in the nonlinear transport regime. The transitions between the levels of the passive dot reduce the drag current and lead to negative differential conductance. We also discuss the dependence on temperature and the role of the lead-dot coupling.
\end{abstract}

Copyright (C) EPLA, 2009

Introduction. - The Coulomb interaction between nearby mesoscopic conductors can be used to control or to detect the charge flow through one system by simply tuning the suitable parameters of the other one. Experimental realizations of this idea revealed new effects or confirmed fundamental concepts in mesoscopic transport: charge sensing [1], real-time detection [2] and controlled dephasing [3]. In a typical setup a quantum point contact (QPC) detector is subjected to a finite bias and due to the charge sensing effect the measured current is shown to depend on the plunger gate voltage applied on a nearby quantum dot. The dot is either coupled to the leads or embedded in a mesoscopic ring in which case the detector provides a "which-path" information. The key mechanism behind these findings is the charge sensing effect: electrons in the detector are sensitive to the electrostatic potential generated by the charges localized in the quantum dot. The potential itself changes in a transport process.

Onac et al. [4] proposed the opposite setting, using the quantum dot as a detector and the nearby QPC as a shot noise generator. It was shown that when the QPC is driven out of equilibrium by applying a bias voltage the Coulomb blockade in the $\mathrm{QD}$ is removed and a dc current flows through it. The mechanism behind this observation relies on the energy absorbsion from the QPC. This process allows the ground-state electrons in the Coulomb-blockaded dot to escape into the leads. In a recent paper McClure et al. [5] replaced the QPC by a quantum dot and investigated the complex pattern of cross current-correlations for a parallel double-dot structure in a four-lead geometry. More precisely, the dots are placed in the Coulomb-blockade regime and two plunger gate voltages are individually varied. It was shown that the interdot Coulomb interaction leads to a complex pattern of the current fluctuations. Goorden and Büttiker [6] proposed a scattering formulation of transport for two interacting conductors by introducing a two-particle $S$ matrix and calculated the cross-correlation function using the leading-order term in the interdot interaction strength, while the intradot interaction is neglected. Also, Haupt et al. [7] recently reproduced qualitatively the results in ref. [5] within the real-time diagrammatic technique.

At the theoretical level the interest in the transport properties of parallel quantum dots (PQD) is not new and has been mainly focused on Kondo correlations and interference effects [8-12]. In most cases the dots accommodate at most two spins, are coupled to the same pair of leads and the driving bias is fixed. On the other hand, in the setup of ref. [5] each dot is coupled to two leads and the bias applied on each dot can be individually varied.

The aim of this work is to investigate one further aspect of electronic transport in Coulomb-coupled parallel quantum dots. Suppose that in the setup of ref. [5] one of the dots (say $\mathrm{QD}_{1}$ ) is weakly coupled to unbiased leads. What happens when the bias applied on the second dot increases? Could one record a steady-state current in $\mathrm{QD}_{1}$ and if so, how it depends on the bias applied on $\mathrm{QD}_{2}$ or on 


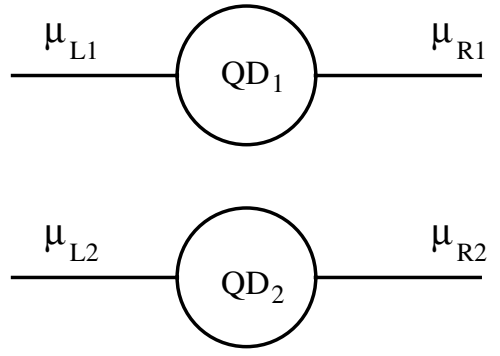

Fig. 1: A sketch of the parallel double-quantum-dot system. There is no tunneling between the dots but they influence each other through the long-range Coulomb interaction of strength $U$.

the level structure of the two dots? This problem is naturally related to the well-known Coulomb drag effect in two-dimensional layers or one-dimensional quantum wires (see the reviews $[13,14])$. The inelastic Coulomb scattering processes imply a momentum transfer between the two subsystems. As a consequence, one can measure a nonvanishing drag voltage in the "passive" system as the electrons pass through the "active" system under the action of a bias. In the theoretical approaches a linear response formula for the drag resistivity is written down while the inelastic effects are calculated up to the second order in the interaction strength. The effective interlayer interaction is computed within different approximations that were recently compared (see ref. [15] and references therein). Mesoscopic Coulomb drag was discussed by Mortensen et al. [16] and by Narozhny et al. [17] and calculations up to third order in the interlayer interaction have also been presented by Levchenko and Kamenev [18]. In the context of Coulomb-coupled quantum point contacts it was argued [19] that a drag current appears either due to the electron-hole asymmetry in the linear regime or due to the shot noise generated by the drive QPC in the non-linear regime.

In this letter we complement the existing thoretical studies by considering two quantum dots in a parallel configuration. We consider both intradot and interdot interactions and compute the steady-state currents in the dots within the Keldysh formalism (see [20-22]) by using the random-phase approximation (RPA) described in ref. [23].

The model. - The two non-interacting quantum dots and the four leads that carry the currents are described by tight-binding Hamiltonians $H_{i} \quad(i=1,2)$ and $H_{L}$ (see also the sketch in fig. 1). The lead-dot coupling $H_{T}$ and the Coulomb interaction $H_{I}$ are included adiabatically through a smooth switching function $\chi(t)$. We denote the on-site energies of $\mathrm{QD}_{i}$ by $\epsilon_{m}^{(i)}$ and the creation/annihilation operators by $c_{m_{i}}^{\dagger} / c_{m_{i}}$. The leads are modelled as one-dimensional semi-infinite discrete chains their sites being indexed by $q_{l}$ where $l=L 1, R 1, L 2, R 2$. The associated creation/annihilation operators are denoted by $d_{q_{l}}^{\dagger}$ and $d_{q_{l}}$. We then have

$$
\begin{aligned}
H(t)=H_{1} & +H_{2}+H_{L}+\chi(t)\left(H_{I}+H_{T}\right) \text { where } \\
H_{i} & =\sum_{i=1}^{2} \sum_{m, n \in Q D_{i}}\left(\epsilon_{m}^{(i)} \delta_{m n}+t_{m n}^{(i)}\right) c_{m_{i}}^{\dagger} c_{n_{i}}, \\
H_{T} & =\sum_{i, n} \sum_{l, q}\left(V_{n_{i} q_{l}} c_{n_{i}}^{\dagger} d_{q_{l}}+h . c .\right) \\
H_{I} & =\sum_{i, j} \sum_{m, n} W_{0, n_{i} m_{j}} c_{n_{i}}^{\dagger} c_{n_{i}} c_{m_{j}}^{\dagger} c_{m_{j}}
\end{aligned}
$$

The matrix element $V_{n_{i} q_{l}}$ stands for the lead-dot hopping integrals and $W_{0, n_{i} m_{j}}=U /\left|r_{n}^{(i)}-r_{m}^{(j)}\right|$ is the bare interaction potential depending on the constant $U$ and on the distance between two sites from the double-dot system. We consider a nearest-neighbor coupling between the leads and the dots and then $V_{n_{i} q_{l}}$ has only four non-vanishing elements that are denoted as $V_{L 1(R 1)}$ and $V_{L 2(R 2)}$. Also, $t_{m n}^{(i)}=t_{D}$, if $m, n$ are nearest neighbors and zero, otherwise. The leads are characterized by their chemical potential, and their temperature included through the Fermi functions. One may write the steady-state current that enters $\mathrm{QD}_{1}$ from the left lead $\left(t_{L}\right.$ is the hopping energy on leads):

$$
\begin{aligned}
J_{L 1}= & \frac{e}{h} \int_{-2 t_{L}}^{2 t_{L}} \mathrm{~d} E \operatorname{Tr}\left\{\Gamma_{L 1} G^{R} \Gamma_{R 1} G^{A}\left(f_{L 1}-f_{R 1}\right)\right. \\
& \left.-\Gamma_{L 1} G^{R} \operatorname{Im}\left(\Sigma_{I}^{<}+2 f_{L 1} \Sigma_{I}^{R}\right) G^{A}\right\} .
\end{aligned}
$$

Evidently, the current entering the dot from the right lead equals $J_{L 1}$ up to a sign $J_{L 1}=-J_{R 1}$. The details of the derivations can be found in ref. [24]. From eq. (4) one observes that even in the absence of a bias on $\mathrm{QD}_{1}$ one is left with the second term in the current formula which is given by the imaginary part of the interaction self-energy. The net current in the passive dot is given precisely by this term which embodies inelastic Coulomb scattering processes which are known to be responsible for the Coulomb drag. The Green functions in eq. (4) are given by the Dyson equation $G=G_{0}+G_{0}\left(\Sigma_{L}+\Sigma_{I}\right) G$ where $G_{0}$ is the Green function of the disconnected noninteracting system and $\Sigma_{I, L}$ are the interaction self-energy and the leads' self-energy. $\Gamma_{L 1}=\Gamma_{R 1}$ are matrices that contain the coupling to the leads and the density of states at the endpoint of the lead. The trace means a sum over the sites of $\mathrm{QD}_{1}$. Since the RPA scheme used to compute $\Sigma_{I}$ was presented in detail in ref. [23] here we only state the main results (see also ref. [25]). The Green function of the non-interacting dots in the presence of the leads is denoted by $G_{\text {eff }}=G_{0}+G_{0} \Sigma_{L} G_{\text {eff }}$ and is used to compute the contour-ordered polarization operator $\Pi_{m n}\left(t_{1}, t_{2}\right)=$ $-G_{\text {eff, } m n}\left(t_{1}, t_{2}\right) G_{\text {eff, } n m}\left(t_{2}, t_{1}\right)$.

We construct then screened interaction $W$, with the retarded, lesser and greater components given by the 
Dyson and Keldysh equations ${ }^{1}$ :

$$
\begin{gathered}
W^{R}(E)=W_{0}+W_{0} \Pi^{R}(E) W^{R}(E), \\
W^{<,>}(E)=W^{R}(E) \Pi^{<,>}(E) W^{A}(E) .
\end{gathered}
$$

The next step consists in calculating the lesser and greater components of the interaction self-energy $\Sigma^{<,>}$. Then the imaginary part of the retarded correlation self-energy is given as $\operatorname{Im} \Sigma_{C}^{R}=\left(\Sigma^{>}-\Sigma^{<}\right) / 2 i$, while its real part should be computed from the Kramers-Kronig identity. The Hartree and exchange contributions are also computed and included in the standard form of the interaction selfenergy $^{2}$ :

$$
\Sigma_{I}=\Sigma_{H}+\Sigma_{X}+\Sigma_{C}
$$

Note that i) $\Sigma_{H}$ does not contribute to the second term in the current since it has no imaginary part; ii) in the $G_{0} W$ approximation $\Sigma_{X}$ cannot couple sites from different dots because the noninteracting Green function has a block-diagonal form; iii) the imaginary part of $\Sigma_{I}$ is non-vanishing as long as one has an applied bias on $\mathrm{QD}_{2}$ in which case $\Sigma_{I}$ is a non-equilibrium quantity - see ref. [24]. The effect of the interdot interaction is then expected to come from higher-order diagrams containing the electron-hole bubble.

The lesser Green function can be used to compute the occupation number $N_{i}$ of each dot or the density of states (DOS) $\rho_{i}$, according to the definitions

$$
N_{i}=\frac{1}{2 \pi} \sum_{m \in \mathrm{QD}_{i}} \int_{-2 t_{L}}^{2 t_{L}} \mathrm{~d} E \operatorname{Im} G_{m m}^{<}=\int_{-2 t_{L}}^{2 t_{L}} \mathrm{~d} E \rho_{i}(E) .
$$

In this work we consider only the long-range Coulomb interaction and disregard the on-site Hubbard interaction as well as the spin degree of freedom.

Results and discussion. - We present below numerical simulations for identical dots having three sites (levels). $\mathrm{QD}_{1}$ is set in the Coulomb blockade regime by taking a small coupling to the leads and by fixing the chemical potentials of the latter such that they do not align with any of its levels. We shall consider two values of $\mu_{0}$ : i) $\mu_{0}=1$ (configuration A) for which the highest level of $\mathrm{QD}_{1}$ is above $\mu_{0}$; ii) $\mu_{0}=2.25$ (configuration $\mathrm{B}$ ), in which all the levels are below $\mu_{0}$ (see the inset in fig. 2(a)). The bias $e V_{2}=\mu_{L 2}-\mu_{R 2}$ is changed by keeping $\mu_{L 2}=3$ and by lowering the chemical potential of the right lead from $\mu_{R 2}=3$ to $\mu_{R 2}=-3$. Therefore the three levels of the active dot will enter the bias window one by one, which is similar to opening more conduction channels in a quantum point contact. The hopping energy $t_{D}$ of the dots is

\footnotetext{
${ }^{1}$ All quantities are finite rank matrices since the leads' selfenergy has non-vanishing elements only at the contact sites while the interaction self-energies are also restricted to sites from the dots.

${ }^{2}$ The explicit expressions can be traced from ref. [23]: $\Sigma^{<,>}$are given in eqs. (21)-(23), while the exchange and Hartree contributions are given by eq. (25) and (26). Note that the first order self-energies do not have lesser/greater components.
}
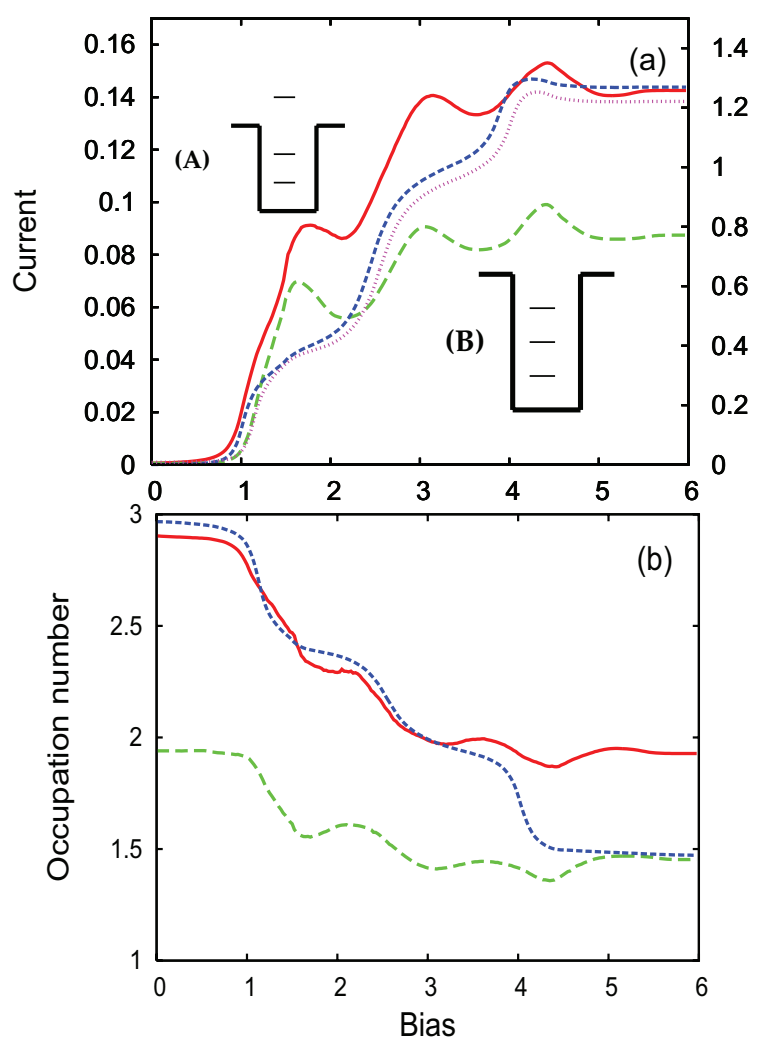

Fig. 2: (Color online) (a) The currents through the dots as a function of bias applied on the active dot. Full line: $J_{L 1}$ (configuration A), long-dashed line: $J_{L 1}$ (configuration B), dashed line: $J_{2}$ (configuration A), dotted line: $J_{L 2}$ (configuration B). The 3rd level in configuration $\mathrm{A}$ is above the chemical potential of the leads. (b) The occupation numbers of the dots as a function of $V_{2}$. Full line: $N_{1}$ (configuration B), long-dashed line: $N_{1}$ (configuration A), dashed line: $N_{2}$. Other parameters: $U=0.15, V_{L 1}=V_{R 1}=0.25, V_{L 2}=V_{R 2}=0.5, k T=0.001$.

chosen as the energy unit and the current is given in units of $e t_{D} / \hbar$.

We show and compare in fig. 2(a) the currents $J_{L 1, L 2}$ entering $\mathrm{QD}_{1,2}$ from the left lead (the value of $J_{L 2}$ is to be read on the right axis) in the two configurations defined above. $J_{L 2}$ increases abruptly at three values of the bias (e.g. at $V_{2}=1$ and $V_{2}=2.3$ and $V_{2}=3.7$ ).

The jumps appear when one level of $\mathrm{QD}_{2}$ enters the bias window $\left[\mu_{L 2}, \mu_{R 2}\right]$ and the smooth regions between them turn to steps if the lead-dot coupling is reduced, recovering thus the well-known $I-V$ curve. We observe that a drag current appears in the passive dot when the first level of $\mathrm{QD}_{2}$ enters the bias window (at $V_{2} \sim 0.85$ ). $J_{L 1}$ continues to increase up to $V_{2} \sim 1.6$ and then decreases in the range $[1.6,2.25]$ until the bias window on the active dot covers one more level. The current eventually settles down for $V_{2}>5$. The configuration $\mathrm{A}$ is characterized by a higher value. It is clear that electrons tunneling through the active dot drag a current in the passive dot and that by increasing $V_{2}$ one opens more conduction channels in $\mathrm{QD}_{2}$ enhancing therefore the Coulomb drag in the passive dot. We have checked that the same effect ensues if one varies 
the gate potential applied to the active dot while keeping a small bias window. In that case a peak appears in the current through the passive dot when the gate potential is such that a level of $\mathrm{QD}_{2}$ becomes resonant.

Another interesting feature is that between any two jumps of $J_{L 2}$ the passive dot experiences a negative differential conductance (NDC) regime which leads to a sawtooth behavior. This rather peculiar feature of quantum dot systems was observed in experiments with serially coupled quantum dots and has been theoretically investigated for double dots [26,27]. To our best knowledge, no experimental results of NDC have been reported for parallel quantum dots as we consider here.

The occupation number $N_{1}$ of the passive dot is given in fig. 2(b). In both configurations $N_{1}$ decreases when $J_{L 1}$ increases. In the NDC regime, however, $N_{1}$ increases slightly and then decreases. The occupation number $N_{2}$ of the active dot in configuration $\mathrm{B}$ shows that as $\mu_{R 2}$ crosses one level of $\mathrm{QD}_{2}$ the occupation number decreases from 1 to $1 / 2$ (see the dashed line in fig. $2(\mathrm{~b})$ ).

We shall investigate in the following the role of the bias applied on the active dot on the observed Coulomb drag and the negative differential conductance regime. On the one hand, it is clear that we need a finite bias on the active dot in order to ensure a net flow of electrons which in turn drive the passive dot out of equilibrium. The energy transferred to the passive dot is then determined by the bias window of the active dot and by the number of states contained in it (see, e.g., fig. 2 in ref. [14]) and allows transitions between different levels of the passive dot as well. The main point here is that if this energy matches the gaps $\Delta_{i}=\mu_{0}-E_{i}$ electrons are allowed to tunnel out from the $i$-th level of the passive dot. In order to check this scenario, we analyze the density of states (DOS) of the passive dot in configuration B. Figures 3(a)-(c) show the energy regions with a nonvanishing DOS; the bright traces allow one to locate the levels of the open quantum dot and to monitor their behavior as the bias $V_{2}$ on the active dot is varied. One can see that at $V_{2}=0$ these levels are located around $E_{1}=-0.75, E_{2}=0.55$ and $E_{3}=1.95$.

At $V_{2} \sim 1$ the DOS in fig. 3(a) already starts to decrease because electrons from this level gain already enough energy to escape into the leads. Figures $3(\mathrm{~b})$ and (c) indicate that the lowest levels come also into play at $V_{2} \sim$ 1.4, because the level spacings $E_{1}-E_{2}$ and $E_{2}-E_{3}$ equal the transferred energy. It is important to observe that the transitions $E_{3} \rightarrow E_{2}$ and $E_{2} \rightarrow E_{1}$ coexist and compete with the drag effect, in the sense that the transferred energy is not entirely spent on exciting electrons from the highest level to the leads but also on such intradot transitions. A comparison with figs. 2(a) and (b) reveals that in the range $[1.4: 2.2]$ the current decreases but the occupation number $N_{1}$ is constant; also, the decrease in the DOS in fig. 3(c) appears at $V_{2}=1.4$ and can only be associated with the transition $E_{3} \rightarrow E_{2}$ because this is the only one allowed at that value of $V_{2}$. The NDC regime disappears at $V_{2} \sim 2.2$ because another electron
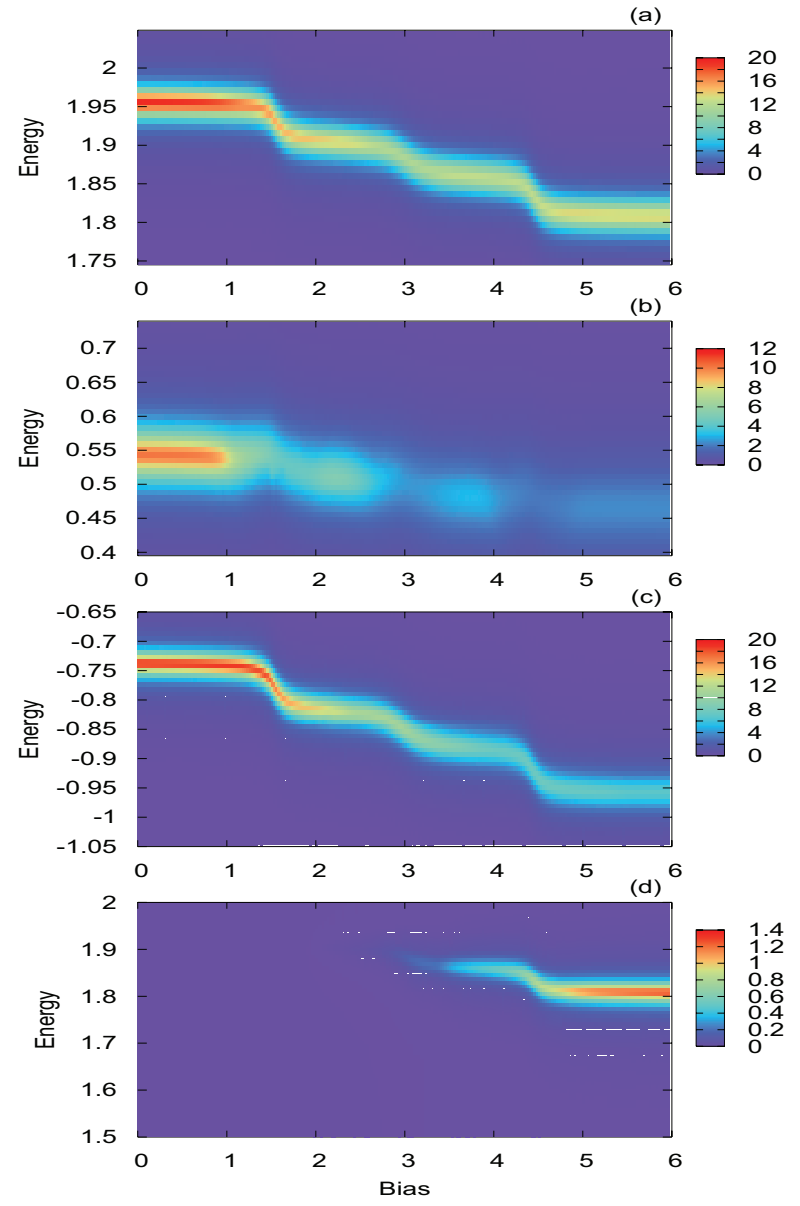

Fig. 3: (Color online) (a)-(c) Relevant regions of the density of states for the passive dot in configuration $\mathrm{B}\left(\mu_{0}=2.25\right)$. (d) The upper level is populated in configuration A even if it is located above the chemical potential of the leads. In this case $\mu_{0}=1$. Other parameters are as in fig. 2 .

enters the bias window of the active dot and the drag effect is enhanced. By further increasing $V_{2}$ the middle level is almost emptied because, on the one hand, it provides electrons into the leads and, on the other hand, the transferred energy exceeds the gap $E_{2}-E_{3}$. Next, at $V_{2}=3$ it is possible to excite electrons from the lowest level directly into the leads and consequently the DOS in fig. 3(c) decreases even more. As expected, the transition $E_{1} \rightarrow E_{3}$ is also allowed; this is confirmed by the slight increase in the DOS in fig. 3(a) and in the occupation number in fig. 2(b). The shifts toward lower energies noticed in the DOS are a consequence of the charge sensing effect: as the occupation numbers of the levels in $\mathrm{QD}_{2}$ decrease from 1 to $1 / 2$ the (upward) Hartree shift they generate in $\mathrm{QD}_{1}$ decreases.

The differences between the drag currents of the two configurations are also explained by considering the sequence of transitions induced by the interdot interaction. In configuration A electrons from the lowest level (which in this case is located around $E_{1}=-0.9$ ) can escape into the leads if $V_{2}=2$ because the chemical potential 


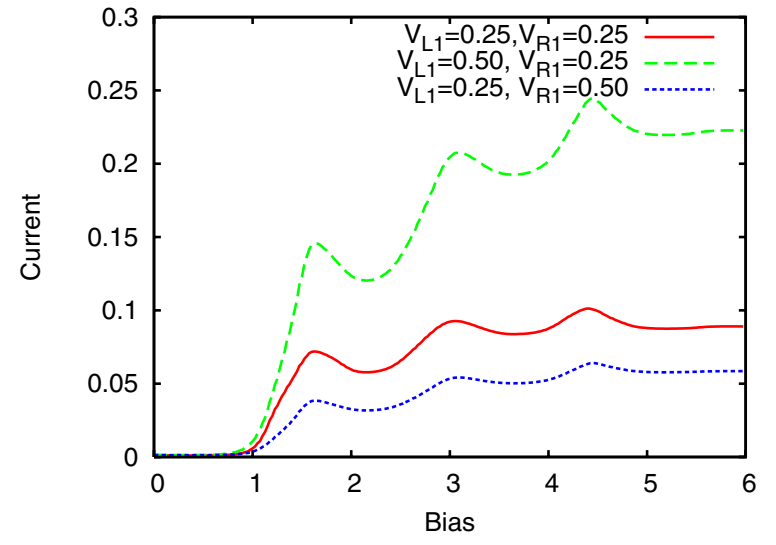

Fig. 4: (Color online) Drag current in configuration A for different values of the coupling strength $V_{R 1}$ and $V_{L 1}$. The regions of negative differential conductance are suppressed at $V_{R 1}=0.5$. This means that the interdot transitions are less important and that electrons rather tunnel out in the leads. Other parameters are $U=0.15, k T=0.001$.

of the leads is $\mu_{0}=1$. As we have seen, this process is not allowed in configuration B until $V_{2}=3$. Figure $3(\mathrm{~d})$ shows the DOS of the passive dot in configuration A for energies above the chemical potential of the leads. One can easily observe that the highest level is populated at large values of the bias, because of the transitions $E_{1} \rightarrow E_{3}$. We have found similar results for other values of the chemical potential and of the interaction strength.

The numerical results in fig. 2(a) clearly show that the inelastic scattering generates a positive Coulomb drag. This is not a universal feature as negative Coulomb drag has been reported in several experiments [28-30]. In these cases the mechanism seem to be quite different from the one we study here, since one varies a magnetic field that induces fluctuations in the charge density. Nevertheless, in quantum dot systems the configuration details can lead to rectification effects that in the problem at hand would add another asymmetry to the effective Coulomb asymmetry. We present in fig. 4 results for asymmetric coupling to the leads. We consider two asymmetric configurations: $V_{L 1}=$ $0.5, V_{R 1}=0.25$ and $V_{L 1}=0.25, V_{R 1}=0.5$ and compare them with the symmetric case $V_{L 1}=V_{R 1}=0.25$. For both cases the drag current is still positive but its amplitude is very sensitive to the asymmetry of the coupling. Clearly the drag current increases when $V_{L 1}$ increases; in contrast, it decreases when $V_{R 1}$ increases. These data suggest that the asymmetry of the coupling works against the Coulomb driving when the coupling to the right lead increases. We propose the following physical picture behind these results. As we have shown, the mechanism of the Coulomb drag gives a net current which flows from the left lead towards the sample $J_{L 1}>0$, or equivalently, from the sample towards the right lead. This means that the in-tunneling current from the left lead $J_{L 1}^{\text {in }}$ exceeds the out-tunneling current $J_{L 1}^{\text {out }}$. Similarly, $J_{R 1}^{\text {out }}$ exceeds $J_{R 1}^{\text {in }}$. We recall that in the Keldysh formalism one computes net steady-state

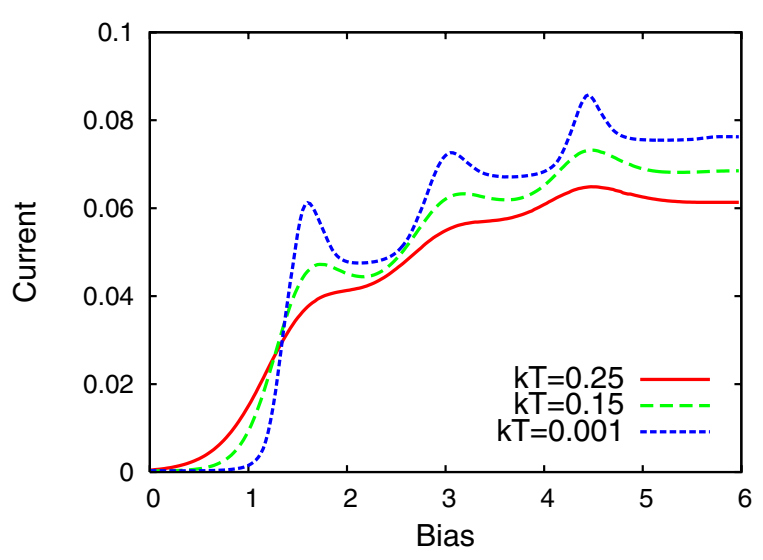

Fig. 5: (Color online) The current through the passive dot in configuration $\mathrm{B}$ as a functions of the bias $V_{2}$ at different temperatures. Passive dot in configuration B. $V_{L 1}=V_{R 1}=0.25$, $U=0.1$.

currents, that is $J_{L 1}=-J_{R 1}=J_{R 1}^{\text {out }}+J_{R 1}^{\text {in }}$. The Coulomb drag is controlled on one hand by the interdot interaction and by the applied bias on the active dot. On the other hand, by increasing $V_{L 1}$ more electrons can enter the dot from the left lead, $J_{L 1}^{\text {in }}$ increases and since it has the same sign as the Coulomb drag the latter will increase as well. In contrast, when $V_{R 1}$ increases from 0.25 to 0.5 electrons enter more easily from the right lead and the in-tunneling current increases. But $J_{R 1}^{\text {in }}$ flows against the Coulomb drag and then the net current decreases as well.

Note that in all cases the drag appears at the same value of $V_{2}$ since $J_{2}$ depends very weakly on $V_{L / R, 1}$ (that is, the jumps between the steps in $J_{2}$ are stable against $V_{L / R, 1}$ ). In a recent experiment of Khrapai et al. [31] a counterflow of electrons was obtained in Coulomb-coupled quantum point contacts; the authors suggested that this feature relies on the acoustic phonons predominantly generated in the drain lead of the active system. In the present approach the role of the acoustic phonons in the leads is not included so we are not able to reproduce their result. Note however that the quantum point constrictions used in their experiment are not set in the Coulomb blockade so the physics could be very different.

As for the temperature effects we find that if $k T \ll U$ there are no significant changes in the drag current. In contrast, when $k T \sim U$ the onset of the drag current is not steep and its slope softens as $k T$ increases. This happens because at higher temperatures the steps in the $I-V$ curve of the active dot are no longer well defined. Electrons can enter the bias window before the levels align with the chemical potential of the right lead. The NDC regions become also flat, and the difference between local minima and maxima in the drag current decreases. We understand these features considering the smearing of the Fermi function, i.e., electrons incident from the left lead are more likely to have lower energy such that the momentum transferred to the passive dot decreases. Temperature effect on the current through the passive dot is illustrated in fig. 5 for configuration B. 
When $\mathrm{QD}_{1}$ is submitted to a bias while $\mathrm{QD}_{2}$ becomes the passive system we obtain similar results; this is a reminiscence of the Onsager relation (see, e.g., [19]).

By taking a lattice constant of few nms and by using the electron effective mass in GaAs for the hopping parameter $t_{D}=\hbar^{2} / 2 m^{*} a^{2}$, we find that the drag current reaches tenths of $\mu A$ for a bias less than $1 \mathrm{~V}$. These parameters are clearly in the experimental range $[28,32]$. We would like to point out that the configurations $\mathrm{A}$ and $\mathrm{B}$ we have discussed here can be met in experiments: by looking at Coulomb diamonds characterizing the passive system one infers information about its spectral structure (location of levels, level spacing, detuning for double dots etc.) and then a specific configuration is chosen for the transport setup.

Conclusions. - We have presented theoretical calculations supporting the existence of a positive Coulomb drag effect in parallel quantum dots. The intradot transitions become possible when the transferred energy matches the level spacing and compete with the drag effect. In this regime the current in the passive dot decreases with the bias. We showed that in the PQD system the level structure determines the behavior of the drag current. This is clearly not the case in larger systems where the spectrum is dense and the coupling to the leads is not weak ${ }^{3}$. The interdot distance in PQD system is very small so that the Coulomb drag effect should be easier to observe in transport measurements with such structures. Since Coulomb drag measurements in 1D wires with embedded dots were already reported [28] and bilayer systems of self-assembled quantum dots are being currently studied [32] we hope the present calculation will motivate further experiments.

$$
* * *
$$

This work is supported by TUBITAK (No. 108T743) and TUBA. VM acknowledges the hospitality of the Physics Department in Bilkent University and the support from the PNCDI2 programme (Grant ID899) and TUBITAK-BIDEP.

\section{REFERENCES}

[1] Johnson A. C., Marcus C. M., Hanson M. P. and Gossard A. C., Phys. Rev. Lett., 93 (2004) 106803.

[2] Elzerman J. M., Hanson R., Willems van Beveren L. H., Witkamp B., Vandersypen L. M. K. and Kouwenhoven L. P., Nature, 430 (2004) 431.

[3] Buks E., Schuster R., Heiblum M., Mahalu D. and UmAnsky V., Nature (London), 391 (1998) 871.

\footnotetext{
${ }^{3}$ The RPA scheme we implement contains a screened interaction and some effects could be observed for larger dots. Unfortunatelly the size of the systems we are able to treat is limited by the computational time and power. The problem is that one needs a dense energy grid for the various integrals we solve numerically in order to get a good resolution of the peaks in the Green functions of the two dots. The number of peaks and their sharpness increase with the number of sites.
}

[4] Onac E., Balestro F., van Beveren L. H. Willems, Hartmann U., Nazarov Y. V. and Kouwenhoven L. P., Phys. Rev. Lett., 96 (2006) 176601.

[5] McClure D. T., DiCarlo L., Zhang Y., Engel H.-A., Marcus C. M., Hanson M. P. and Gossard A. C., Phys. Rev. Lett., 98 (2007) 056801.

[6] Goorden M. C. and Büttiker M., Phys. Rev. B, 77 (2008) 205323; Phys. Rev. Lett., 99 (2007) 146801.

[7] Haupt S., Aghassi J., Hettler M. H. and Schön G., arXiv:0802.3579.

[8] Tanaka Y. and Kawakami N., Phys. Rev. B, 72 (2005) 085304.

[9] Lara G. A., Orellana P. A., Yañez J. M. and Anda E. V., Solid State Commun., 136 (2005) 323.

[10] Sztenkiel D. and Swirkowicz R., J. Phys.: Condens. Matter, 19 (2007) 176202.

[11] Zitko R. and Bonca J., Phys. Rev. B, 74 (2006) 045312.

[12] Trocha P. and Barnas J., Phys. Rev. B, 76 (2007) 165432.

[13] Rojo A. G., J. Phys.: Condens. Matter, 11 (1999) R31.

[14] Debray P., Gurevich V., Klesse R. and Newrock R. S., Semicond. Sci. Technol., 17 (2002) R21.

[15] Asgari R., Tanatar B. and Davoudi B., Phys. Rev. B, 77 (2008) 115301.

[16] Mortensen N. A., Flensberg K. and Jauho A.-P., Phys. Rev. Lett., 86 (2001) 1841; Phys. Rev. B, 65 (2002) 085317.

[17] Narozhny B. N. and Aleiner I. L., Phys. Rev. Lett., 84 (2000) 5383; Narozhny B. N., Aleiner I. L. and Stern Ady, Phys. Rev. Lett., 86 (2001) 3610.

[18] Levchenko A. and Kamenev A., Phys. Rev. Lett., 100 (2008) 026805.

[19] Levchenko A. and Kamenev A., Phys. Rev. Lett., 101 (2008) 216806.

[20] Meir Y. and Wingreen N. S., Phys. Rev. Lett., 68 (1992) 2512.

[21] Jauho A.-P., Wingreen N. S. and Meir Y., Phys. Rev. $B, 50$ (1994) 5528.

[22] Haug H. and Jauho A.-P., Quantum Kinetics in Transport and Optics of Semiconductors (Springer, Berlin) 1996.

[23] Moldoveanu V. and Tanatar B., Phys. Rev. B, 77 (2008) 195302.

[24] Moldoveanu V., Ţolea M. and Tanatar B., Phys. Rev. B, 75 (2007) 045309.

[25] Thygesen K. S. and Rubio A., Phys. Rev. B, 77 (2008) 115333.

[26] Pedersen J. N., Lassen B., Wacker A. and Hettler M. H., Phys. Rev. B, 75 (2007) 235314.

[27] Fransson J. and Eriksson O., J. Phys.: Condens. Matter, 16 (2004) L85.

[28] Shimizu M., Yamamoto M., Stopa M., Honda M. and Tarucha S., Physica E, 26 (2005) 460.

[29] Yamamoto M., Stopa M., TokURA Y., Hirayama Y. and Tarucha S., Science, 313 (2006) 204.

[30] Price A. S., Savchenko A. K., Narozhny B. N., Allison G. and Ritchie D. A., Science, 316 (2007) 99.

[31] Khrapai V. S., Ludwig S., Kotthaus J. P., Tranitz H. P. and Wegscheider W., Phys. Rev. Lett., 99 (2007) 096803.

[32] Barthold P., Hohls F., Maire N., Pierz K. and Haug R. J., Phys. Rev. Lett., 96 (2006) 246804. 